\title{
A Total Synthesis of the Antifungal Deoxyaminocyclitol Nabscessin B from L-(+)-Tartaric Acid
}

\author{
Xiang Ma, Qiao Yan, Martin G. Banwell* and Jas S. Ward \\ Research School of Chemistry, Institute of Advanced Studies \\ The Australian National University, Canberra, ACT 2601, Australia.
}

Supporting Information Placeholder<smiles>O=C(O)[C@H](O)[C@@H](O)C(=O)O</smiles>

$L-(+)$-tartaric acid

(3)

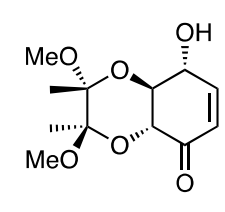

4

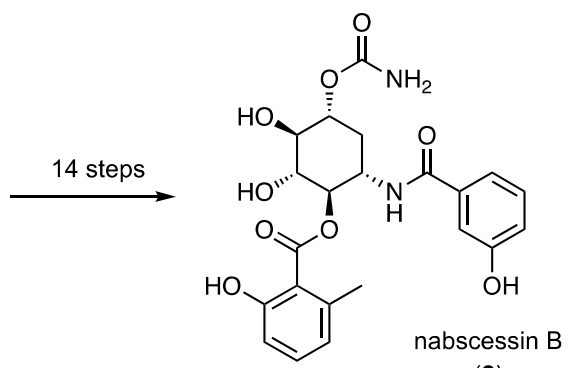

(2)

ABSTRACT: Aminocyclitol 2, a recently isolated and notable anti-fungal agent, was prepared from homochiral $\gamma$ hydroxycyclohexenone $\mathbf{4}$ that is itself available in six steps from $L-(+)$-tartaric acid (3). The well-defined rigidifying effect arising from the 1,2-diacetal protecting group associated with compound 4 and its derivatives allows for high levels of regio- and stereo-chemical control in the manipulation of the cyclitol framework.

Recently, Ishibashi and co-workers reported ${ }^{1}$ the isolation of the deoxyaminocyclitols 1 and 2 (Figure 1) from the culture broths of the pathogenic actinomycete Nocardia abscessus IFM $10029^{\mathrm{T}}$, a species derived from an intraarticular abscess associated with the knee of a human patient. Compounds 1 and $\mathbf{2}$ were named nabscessions $\mathrm{A}$ and $\mathrm{B}$, respectively, and the illustrated and isomeric structures were established through extensive spectroscopic analyses, most notably 2D NMR studies. Both nabscessins embody a 2-deoxy-scyllo-inosamine core. ${ }^{2}$
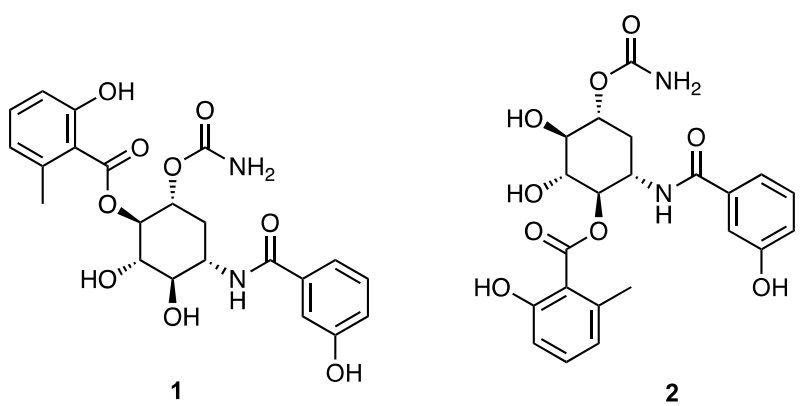

Figure 1: The structures of the aminocyclitols nabscessins A (1) and B (2)

A preliminary biological evaluation of compounds $\mathbf{1}$ and 2 revealed that they act against Cryptococuss neoformans with $\mathrm{IC}_{50}$ values of 32 and $16 \mu \mathrm{g} / \mathrm{mL}$, respectively $(C$. neoformans is a ubiquitous and encapsulated yeast that has been a significant cause of mortality in immune- compromised patients, especially those suffering from AIDS $\left.^{3}\right)$.

The novel structures associated with natural products 1 and 2 together with the ongoing interest in the development of selective approaches to variably functionalized aminocyclitols and their deployment as anti-infective agents ${ }^{4}$ prompted us to develop routes to these compounds. We now report a synthesis of nabscessin B (2) from $L-(+)$-tartaric acid that serves to confirm its structure, including its absolute stereochemistry.

We have recently described the generation of various homochiral cyclitols from either the (+)- or (-)-forms of tartaric acid and deployed these in the preparation of the fungal metabolite aspergillusol B and certain analogues of the alkaloid galanthamine. ${ }^{5}$ As revealed here, one of these same cyclitols has served as a precursor to the title alkaloid. The synthetic sequence leading to a deoxyinositol precursor of nabscessin B (2) is shown in Scheme 1. Thus, as previously reported, 5a $L$-(+)-tartaric acid (3) was converted over six steps, including those involving vinylation and ring-closing metathesis reactions, into the 1,2diacetal-containing ${ }^{6} \gamma$-hydroxy-cyclohexenone 4. Protection of the hydroxyl group within this last compound under standard conditions gave the tert-butyldiphenylsilyl (TBDPS) ether 5 (98\%) that was itself subjected to Luche reduction and so affording, stereoselectively, the protected conduritol 6 (95\%). Hydroxyl-directed epoxidation of this allylic alcohol using $m$-chloroperbenzoic acid ( $m$ CPBA) then gave the epoxy-alcohol 7 (85\%). Treatment of compound 7 with sodium borohydride in the presence of 
boron trifluoride diethyl etherate ${ }^{7}$ resulted in preferential trans-diaxial and thus regio-selective cleavage of the oxirane ring to give a chromatographically separable mixture of the deoxyinositols 8 (15\%) and $\mathbf{9}(85 \%)$. The structure of these products follows from both the derived spectroscopic data and the single-crystal X-ray analyses of two derivatives of the latter compound as discussed below.

Scheme 1: Synthesis of the deoxyinositol 9

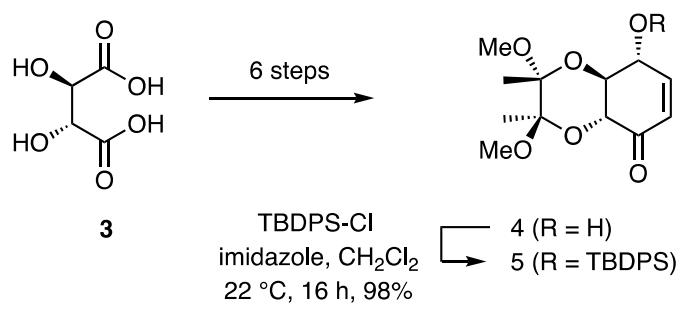

$\mathrm{NaBH}_{4}, \mathrm{CeCl}_{3}$
$\mathrm{CH}_{3} \mathrm{OH}, 0{ }^{\circ} \mathrm{C}$
$0.5 \mathrm{~h}, 95 \%$

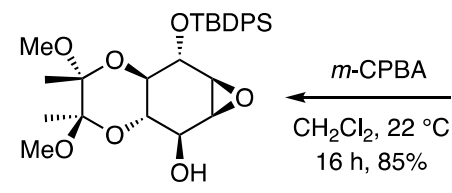

7
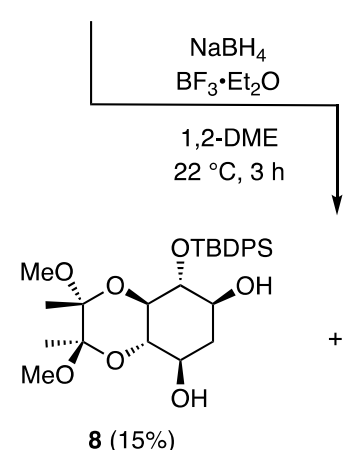

$8(15 \%)$

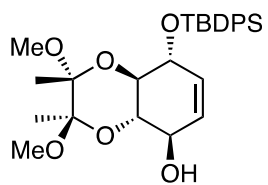

6

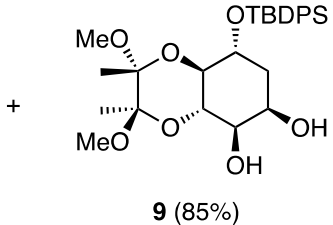

The synthetic sequence used for effecting the conversion of compound 9 into the 2-deoxy-scyllo-inosamine core of target $\mathbf{2}$ is shown in Scheme 2 . Thus, the equatorial hydroxyl group within diol $\mathbf{9}$ was selectively protected as the corresponding $p$-methoxybenzyl (PMB) ether through treatment with $\mathrm{PMB}-\mathrm{Cl}$ in the presence of $n-\mathrm{Bu}_{2} \mathrm{SnO}$ and tetra- $n$-butylammonium iodide (TBAI) ${ }^{8}$ This was followed by triflation of the remaining and axial alcohol using triflic anhydride in the presence of pyridine and thus giving ester 10 in $81 \%$ yield over the two steps involved. Reaction of a solution of the last compound in DMF with sodium azide resulted in the rapid displacement of the axially oriented triflate moiety and formation of the azido-substituted cyclitol $\mathbf{1 1}$ (91\%), the structure of which was confirmed by single-crystal X-ray analysis [see the Supporting Information (SI) for details]. Staudinger reduction of compound $\mathbf{1 1}$ using triphenylphosphine in the presence of water then gave the corresponding amine that was immediately coupled with 3(methoxymethoxy)benzoic acid 9 in the presence of $N$ [(dimethylamino)-1 $H-1,2,3$-triazolo-[4,5-b]pyridine-1methyle ne]- $N$-methylmethanaminium hexafluorophosphate $\mathrm{N}$-oxide (HATU) ${ }^{10}$ and $\mathrm{N}, \mathrm{N}$-di-isopropylethylamine (DIPEA) and thus affording amide 12 (81\%). All of the spectral data acquired on compound $\mathbf{1 2}$ were in complete accord with the assigned structure with the most diagnostic features being the appearance of a resonance due to an amide carbonyl carbon at $\delta_{\mathrm{C}} 166.8$ in the ${ }^{13} \mathrm{C}$ NMR spectrum and the presence N-H and amide carbonyl stretching bands at 3296 and $1640 \mathrm{~cm}^{-1}$ in the infra-red spectrum.

Scheme 2: Elaboration of the deoxyinositol 9 to the 2deoxy-scyllo-inosamine core of target 2

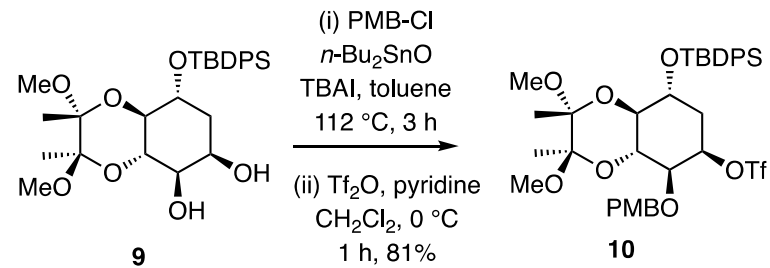

$1 \mathrm{~h}, 81 \%$

10
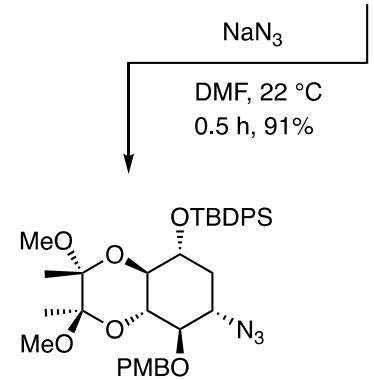

11

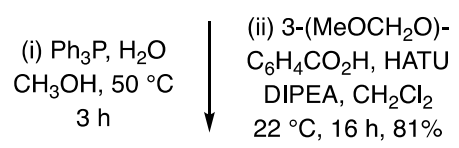

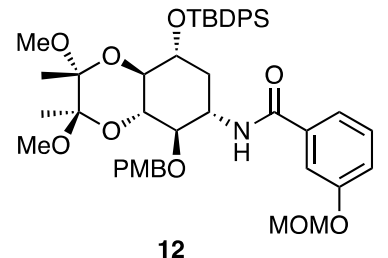

The next stage of the synthesis of nabscessin B (2) is shown in Scheme 3 and involved the successive cleavage of the PMB and TBDPS ether moieties associated with compound 12 using 2,3-dichloro-5,6dicyanobenzoquinone (DDQ) then tetra- $n$ butylammonium fluoride (TBAF) and thus affording the diol 13 in $93 \%$ yield. The structure of compound 13 was confirmed by single-crystal X-ray analysis (see SI for details). Selective esterification of the equatorially oriented and $\beta$-configured hydroxyl group over its $\alpha$-oriented counterpart on the opposite side of the cyclohexane ring was achieved using commercially available 6methylsalicylic acid in the presence of $\mathrm{N}-3^{-}$ 
dimethylaminopropyl)- $N^{\prime}$-ethylcarbodiimide (EDC) then afforded compound 14 (6o\%). This was accompanied by ca. $18 \%$ of the corresponding bis-ester. The structure of compound 14 follows unambiguously from a range of NMR studies. Most particularly, the resonance due to the proton associated with the oxymethine moiety carrying the newly introduced ester group appears as a one-proton triplet $(J=10.2 \mathrm{~Hz})$ at $\delta_{\mathrm{H}} 5.47$ and is vicinally coupled to the multiplet at $\delta_{\mathrm{H}} 4.58$ arising from the proton attached to the ring carbon carrying the amide moiety. The selectivity observed in this reaction is interesting since the notionally more congested hydroxyl group in precursor 13 is esterified. Hydrogen bonding and $\pi$-stacking interactions between the benzamide residue of substrate 13 and the activated form of 6-methylsalicylic acid may be responsible. Treatment of compound 14 with 2,2,2trichloroacetyl isocyanate ${ }^{11}$ in dichloromethane led, after aqueous workup, to carbamate $\mathbf{1 5}(88 \%)$.

Scheme 3: Synthesis of a protected form, 15, of nabscessin B (2)
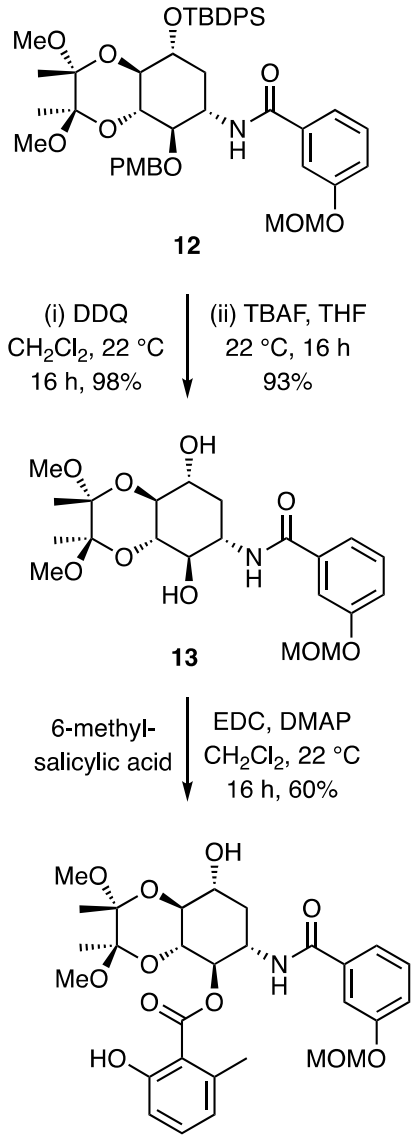

14

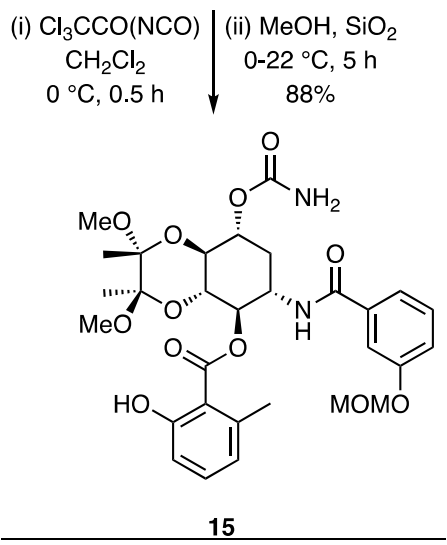

The final step in the total synthesis of the title aminocyclitol involved (Scheme 4) treating compound 15 with a 9:1 $\mathrm{v} / \mathrm{v}$ mixture of trifluoroacetic acid (TFA) and water at 22 ${ }^{\circ} \mathrm{C}$ and this gave, after concentration of the reaction mixture and subjection of the residue to flash column chromatography, nabscessin B (2) as an amorphous powder in $81 \%$ yield. The ${ }^{1} \mathrm{H}$ and ${ }^{13} \mathrm{C}$ NMR, IR and mass spectral data obtained on this material were consistent with the assigned structure and matched those reported for the natural product (see the SI for a tabular comparison of the two sets of ${ }^{1} \mathrm{H}$ and ${ }^{13} \mathrm{C}$ NMR spectroscopic data). The specific rotation of the synthetic material was somewhat higher than that reported for the natural product $\left\{[\alpha]_{\mathrm{D}}\right.$ $+21.3(c$ 1.5, MeOH $)$ vs. $[\alpha]_{\mathrm{D}}+15.7($ c 1.o, $\left.\mathrm{MeOH})\right\}$. The origins of this difference are not entirely clear but a possible 
explanation is that the natural product contains a levorotatory impurity. Nevertheless, the work reported here serves to confirm the illustrated structure of nabscessin B, including its absolute stereochemistry.

Scheme 4: The deprotection of compound 15 to form nabscessin B (2)

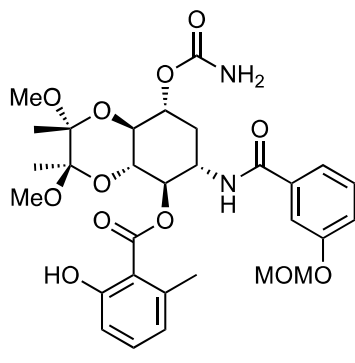

15

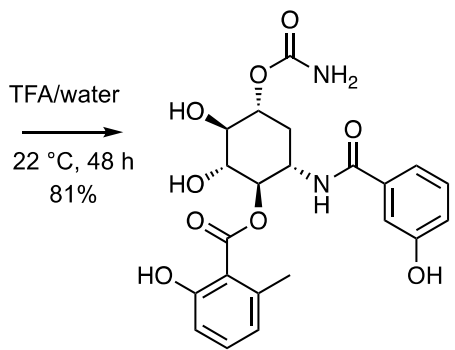

2
The protocols defined above provide a distinct new route to aminocyclitols in that they do not start from an inositol, rather the cyclitol framework is constructed de novo. ${ }^{\mathrm{d}}$ Given that both enantiomeric forms of tartaric acid are readily obtained (each at a modest price), a significant range of aminocyclitols will be available using various broadly applicable modifications of the methods detailed above.

\section{ASSOCIATED CONTENT}

\section{Supporting Information}

Experimental procedures, spectroscopic data, copies of the NMR spectra of compounds 5-15 and 2 together with the X-ray data and derived ORTEP for compounds $\mathbf{1 1}$ and 13. The Supporting Information is available free-of-charge on the ACS Publications website at DOI: 10.1021/acs.orglett.XXXXXX.

\section{AUTHOR INFORMATION}

\section{Corresponding Author}

* E-mail: Martin.Banwell@anu.edu.au

\section{Author Contributions}

The manuscript was written through the contributions of all the authors. All authors have given approval to the final version of the manuscript.

\section{Notes}

The authors declare no competing financial interest.

\section{ACKNOWLEDGEMENTS}

We thank the Australian Research Council and the Institute of Advanced Studies for financial support. XM is the grateful recipient of a GEP Fellowship provided by the Guangzhou Municipal Government while QY acknowledges the provision of support from the CSC of the People's Republic of China. The assistance of Dr Jingkun Fang (Nanjing University of Science and Technology) in pre- paring some key intermediates is gratefully acknowledged.

\section{REFERENCES}

1. Hara, S., Ishikawa, N., Hara, Y.; Nehira, T., Sakai, K., Gonoi, T., Ishibashi, M. J. Nat. Prod., 2017, 8o, 565 .

2. For a previous study on the synthesis of this framework, see: Yu, J., Spencer, J. B. Tetrahedron Lett., 2001, 42, 4219.

3. Bose, I., Reese, A. J., Ory, J. J., Janbon, G., Doering, T. L. Eukaryotic Cell, 2003, 2, 655.

4 . For a selection of relevant work over the preceding decades, see: (a) Rinehart, K. L., Jr.; Stroshane, R. M. J. Antibiot. 1976, 29, 319; (b) Igarashi, K.; Honma, T.; Fujiwara, T.; Kondo, E. J. Antibiot., 1981, 34, 288; (c) Kühlmeyer, R., Keller, R., Schwesinger, R., Netscher, T., Fritz, H., Prinzbach, H. Chem. Ber., 1984, 117, 1765; (d) Yokoyama, K.; Kudo, F.; Kuwahara, M.; Inomata, K.; Tamegai, H.; Eguchi, T.; Kakinuma, K. J. Am. Chem. Soc., 2005, 127, 5869; (e) Busscher, G. F., Rutjes, F. P. J. T., van Delft, F. L. Chem. Rev., 2005, 105, 775; (f) Coste, G., Horlacher, T., Molina, L., Moreno-Vargas, A. J., Carmona, A. T., Robina, I., Seeberger, P. H., Gerber-Lemaire, S., Synthesis, 2011, 1759; (g) Duscha, S., Boukari, H., Shcherbakov, D., Salian, S., Silva, S., Kendall, A., Kato, T., Akbergenov, R., Perez-Fernandez, D., Bernet, B., Vaddi, S., Thommes, P., Schacht, J., Crich, D., Vasella, A., Böttger, E. C. mBio, 2014, 5, eo1827-14.

5. (a) Buckler, J. N., Schwartz, B. D., Banwell, M. G. Heterocycles, 2017, 95, 290; (b) Buckler, J. N., Taher, E. S., Fraser, N. J., Willis, A. C., Carr, P. D., Jackson, C. J., Banwell, M. G. J. Org. Chem., 2017, 82, 7869; (c) Buckler, J. N., Meek, T., Banwell, M. G., Carr, P. D. J. Nat. Prod., 2017, 8o, 2088. For related earlier work see (d) Jørgensen, M., Iversen, E. H., Paulsen, A. L., Madsen, R. J. Org. Chem., 2001, 66, 4630.

6. For a very useful review on this type of protecting group, see: Ley, S. V., Polara, A. J. Org. Chem., 2007, 72, 5943.

7. For a related conversion, see: Andresen, T. L. Skytte, D. M., Madsen, R. Org. Biomol. Chem., 2004, 2, 2951.

8. Horne, G., Potter, B. V. L. Chem. Eur. J., 2001, 7, 80.

9. Miyakoshi, H., Miyahara, S., Yokogawa, T., Endoh, K., Muto, T., Yano, W., Wakasa, T., Ueno, H., Chong, K. T., Taguchi, J., Nomura, M., Takao, Y., Fujioka, A., Hashimoto, A., Itou, K., Yamamura, K., Shuto, S., Nagasawa, H., Fukuoka, M. J. Med. Chem., 2012, 55, 6427.

10. Carpino, L. E. J. Am. Chem. Soc., 1993, 115, 4397.

11. Ichikawa, Y., Kariya, N., Hasegawa, T. Org. Synth., 2013, 90, 271. 
